\title{
Management of pulmonary artery sling (anomalous left pulmonary artery arising from right pulmonary artery): a conservative approach
}

\author{
P. D. PHELAN AND A. W. VENABLES \\ From the Departments of Thoracic Medicine and Cardiology, Royal Children's Hospital, \\ Melbourne, Australia
}

Phelan, P. D., and Venables, A. W. (1978). Thorax, 33, 67-71. Management of pulmonary artery sling (anomalous left pulmonary artery arising from right pulmonary artery): a conservative approach. An anomalous left pulmonary artery arising from a right pulmonary artery and passing between the trachea and oesophagus was seen in seven patients over a period of 20 years. All of them had main airway obstruction. Surgical division and re-anastomosis of the anomalous artery in front of the trachea produced an unsatisfactory result in the first two patients. The only surgical procedure undertaken in the subsequent five patients was division of a ligamentum arteriosum; this formed part of a compressing ring in one patient. Three patients, one of whom had associated tracheobronchial stenosis, became completely asymptomatic. Two others, both with tracheobronchial stenosis, still have symptoms; the older has shown considerable improvement, while the younger is only 7 months old. It is suggested that a more conservative approach to the management of this anomaly is justified. In patients with associated tracheobronchial stenosis, symptoms are most probably the result of that anomaly. Surgical division of the anomalous artery is unlikely to be beneficial, and death from postoperative complications frequently occurs. Surgical division of the anomalous artery in patients with extrinsic tracheal compression should be considered only if life is threatened, as spontaneous improvement can be anticipated. Recent advances in respiratory care should assist in the conservative management of distressed infants.

Three recent reviews of patients with anomalous left pulmonary artery (pulmonary artery sling) and associated tracheobronchial obstruction (Grover et al., 1975; Koopot et al., 1975; Sade et al., 1975) have stressed the high mortality from this condition and the poor results of surgery. The anomalous left pulmonary artery arises from the posterior aspect of the right pulmonary artery, passes over the right main bronchus near its origin, then turns left to pass between the trachea and the oesophagus to reach the hilum of the left lung at a somewhat lower level than normal (Fig. 1). About half the patients with this anomaly have associated anomalies of the tracheobronchial tree, and a similar percentage have other cardiovascular anomalies.

About one-third of the patients reported died without surgery; most of the remainder have had some surgical procedure, usually division of the anomalous pulmonary artery and reanastomosis in front of the trachea. The operative mortality was about $50 \%$. Only 13 of the 23 survivors of division and re-anastomosis of the anomalous pulmonary artery have been investigated postoperatively. In 11 of them, there was no flow into the left pulmonary artery (Philp et al., 1972; Tesler et al., 1974; Grover et al., 1975; Koopot et al., 1975; Sade et al., 1975).

Because of the unsatisfactory results in the first two patients at the Royal Children's Hospital (Hiller and Maclean, 1957), a conservative approach to the anomalous left pulmonary artery was adopted for the five subsequent patients. It seems of value to document their progress and to suggest an alternative method of management of infants with this anomaly. 


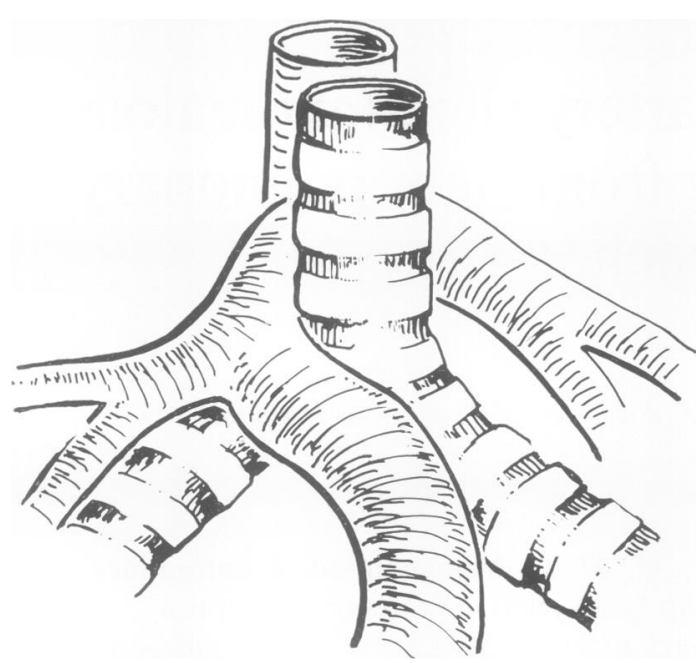

Fig. 1 Schematic diagram showing origin of left pulmonary artery and its aberrant course between the trachea and oesophagus.

\section{Case reports}

CASE 1 (Hiller and Maclean, 1957)

A girl born on 1 April 1953 had respiratory distress from birth manifested by cough, mild inspiratory stridor, and expiratory wheeze. When first seen at the age of 2 years 7 months, she had mild respiratory distress. The diagnosis of anomalous left pulmonary artery was made from a pulmonary angiogram. Operative correction was undertaken at the age of 3 years 2 months with division and re-anastomosis of the left pulmonary artery in front of the trachea. The operation was technically successful but a postoperative angiogram showed complete occlusion of the left pulmonary artery. When last seen aged 12 years, she was asymptomatic with normal exercise tolerance. However, investigation showed no perfusion and minimal ventilation of the left lung.

CASE 2 (Hiller and Maclean, 1957)

From about 6 weeks of age a boy, born on 23 March 1955, had respiratory distress, which was moderate to severe at times. He had an inspiratory stridor and expiratory wheeze. The diagnosis of anomalous left pulmonary artery was made at the age of 23 months from the characteristic anterior indentation seen on a barium swallow and was confirmed by a pulmonary angiogram. Soon after the diagnosis had been established, operative correction was attempted. The pulmonary artery was divided and re-anastomosed in front of the trachea. However, the child died 15 minutes after the conclusion of the operation. At necropsy he had stenosis of the lower trachea.

CASE 3 (Hiller and Maclean, 1957)

A girl, born on 15 December 1956, was first seen at the age of 5 months with a short history of respiratory infection. She had inspiratory stridor and expiratory wheeze and was moderately distressed. A diagnosis of anomalous left pulmonary artery was made from a barium swallow and pulmonary angiogram. After seven weeks in hospital her symptoms improved although stridor and wheeze persisted. This gradually settled over three to four months. By the age of 12 months she had noisy breathing only when upset. From the age of 18 months she had no significant respiratory symptoms.

She was last seen aged 20 years, when her effort tolerance was normal. Over the preceding two years she had developed a productive cough with respiratory infections, but during this period she had been smoking 10-20 cigarettes per day. Lung volumes and maximum expiratory flow volume curve were normal.

CASE 4

A girl, born on 25 May 1959, was first seen at the age of 12 months with a history of noisy breathing since birth. This had increased somewhat over the two months before her first admission to hospital. She had a minor inspiratory stridor and marked expiratory wheeze. She was quite distressed for about four weeks. During her second year of life she had frequent periods of exacerbation of noisy breathing, usually associated with intercurrent respiratory infections. She improved somewhat in her third year but at the age of 4 years she had a further quite severe episode and the diagnosis of anomalous left pulmonary artery was made from a barium swallow and pulmonary angiogram. Bronchoscopic examination showed that the lower half of the trachea was stenosed to about half its normal diameter. The appearance was that of intrinsic stenosis, not external compression. A bronchogram confirmed the tracheal stenosis. No surgical procedures were undertaken. She gradually lost her noisy breathing by the age of 5 and ceased to have worrying episodes of 0 respiratory obstruction. Subsequently she had no respiratory symptoms. When last reviewed at the age of 12 she was totally free of symptoms. Pulmonary function tests showed a slightly reduced vital capacity of 21 (predicted normal $2 \cdot 31$ ), normal total lung capacity, and reduced maximum expiratory flows at high lung volumes on a maximum expiratory flow volume curve (Fig. 2). 

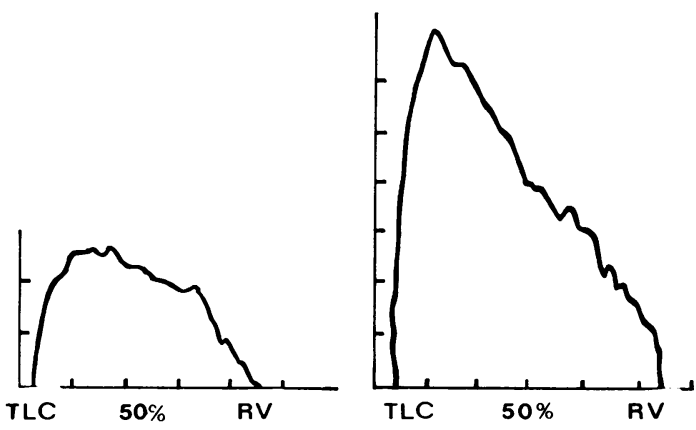

A

respiratory distress. From the age of about 3 years there was slow improvement with a decrease in the amount of stridor and wheeze. Growth was slow. From age 4 years he has required no further admission to hospital, and at age 8 he is well and can keep up with other children. He has minimal expiratory wheeze when excited. A repeat bronchogram when he was 4 years old showed persistence of stenosis of the lower trachea but this was less marked than at the previous examination.

\section{CASE 6}

A girl, born on 23 December 1971, was well until the age of 5 months, when she developed noisy breathing associated with respiratory infection.

Fig. 2 Flow volume curves from $(A)$ case 4 and $(B)$ a healthy girl of similar age and height. Expired volume on $x$-axis, each division representing $0.5 l$, and flow rate on y-axis, each division representing $2.5 l \mathrm{~s}^{-1}$. The peak flow in case 4 was markedly reduced.

\section{CASE 5}

A boy, born on 16 June 1968, was diagnosed as having aplasia of the right lung on the first day of life. About two weeks after birth he developed an inspiratory stridor and expiratory wheeze, which were persistent. He had many periods during which his respirations were distressed. A barium swallow examination was normal. Bronchoscopy showed stenosis of the lower third of the trachea. This finding was confirmed by a bronchogram, which showed that the stenosis extended to the first part of the left main bronchus. A very short, blindly ending right main bronchus was seen. An aortogram suggested that the lower trachea was compressed by a grossly distorted and elongated aortic arch.

At thoracotomy the lower trachea was compressed by a vascular ring. The aortic arch passed to the left of the trachea. The pulmonary artery passed to the right of the trachea, its main left branch arising on the right of the trachea and then passing between it and the oesophagus to reach the left lung. The pulmonary artery and aorta were bound together by the ligamentum arteriosum. When the ligamentum arteriosum was divided, all extrinsic pressure on the trachea appeared to be relieved and no further surgical procedure was undertaken. The stenosis of the lower trachea and the first part of the left main bronchus was confirmed. After operation there was significant improvement although inspiratory stridor and expiratory wheeze continued and the child had frequent admissions to hospital with

The noisy breathing persisted. She had two further periods of severe respiratory distress before being seen first at the age of 8 months.

At that stage she had an inspiratory stridor, expiratory wheeze, and a brassy cough. The diagnosis of anomalous left pulmonary artery was made from a barium swallow and pulmonary angiogram. A tracheogram showed narrowing of the lower third of the trachea, and at bronchoscopy this was considered to be due to posterior extrinsic tracheal compression. She had five further periods of worrying respiratory distress requiring inpatient treatment before the age of 2 years. From age 2 onwards she gradually lost all noisy breathing. She was last seen, aged 5 years, when she had been completely well for the past two years. Her height and weight were both on the 50 th percentile and she had normal effort tolerance. A chest radiograph was normal. It was not possible to carry out pulmonary function tests because of the child's age.

\section{CASE 7}

A boy, born on 1 July 1976, was first admitted to hospital when aged 4 months with a 10-day history of noisy breathing associated with a respiratory infection. He was moderately distressed and had inspiratory stridor and expiratory wheeze. A diagnosis of anomalous left pulmonary artery was made from a barium swallow and pulmonary angiogram. Bronchoscopy showed marked stenosis of the lower third of the trachea and of the origin of the right main bronchus. After bronchoscopy the child had severe respiratory distress for about four days. A bronchographic examination was not undertaken because of the potential risks. $\mathrm{He}$ gradually improved over three weeks and when reviewed a month after discharge from hospital he had only minimal inspiratory stridor and expiratory wheeze. 


\section{Discussion}

The five patients in this series who did not have surgical division of the anomalous left pulmonary artery have survived with either complete resolution (cases 3,4 , and 6 ) or significant improvement (cases 5 and 7) in symptoms of airways obstruction. The only surgical procedure performed in these patients was division of a ligamentum arteriosum to open up a complete vascular ring (case 5). Of the two patients who had surgical division and re-anastomosis of the aberrant artery, one had postoperative thrombosis of the reanastomosed vessel, and the other died immediately after the operation. The surviving patients have been followed for periods ranging from two months to 20 years.

The results indicate that a more conservative approach to the management of patients with anomalous left pulmonary artery is justified. The symptoms of tracheal compression in patients who do not have associated tracheobronchial anomalies decrease progressively without surgical transfer of the abnormally placed vessel. If intrinsic tracheal or bronchial stenosis is present, symptoms of airway obstruction are more likely to result from that, and surgical division of the aberrant artery is not indicated. In fact the only recorded long-term survivors with associated tracheobronchial stenosis are cases 4 and 5 in the present series and another child who had division and posterior anastomosis of the right main stem bronchus (Lochard et al., 1963). Surgical division of the anomalous artery in such patients seems usually to have resulted in death because of postoperative complications. With conservative management symptoms arising from tracheobronchial stenosis diminished as the patients grew, and in case 4 disappeared completely. This patient has physiological evidence of persisting large airway obstruction in that expiratory flow at high lung volumes during a forced expiration was reduced (Miller and Hyatt, 1973). However, this appeared to be of no functional significance.

Koopot et al. (1975) were able to find seven survivors, including case 3 of the present series, who had no specific surgical procedure. However, most of these patients had minimal or no symptoms. While the symptoms in cases 3,4 , and 6 were less than in many of those reported in the literature, they were still sufficient to cause anxiety to parents and to medical attendants. Case 6 had nine admissions to hospital with moderate to severe respiratory distress before she began to improve spontaneously at the age of about 2 years. No patient in this series had hyperinflation of part or the whole of the right lung, which has been a common finding in other reported cases with obstruction of the right main bronchus from an anomalous left pulmonary artery. This radiological finding is probably indicative of severe obstruction.

The long-term effects of the anomalous position of the left pulmonary artery are probably of little importance. Two adults with anomalous left pulmonary artery have been reported. An asymptomatic example was discovered from the chest radiograph performed on a 51-year-old man (Kale et al., 1970). Arteriosclerotic dilatation of the anomalous pulmonary artery produced dysphagia of six months' duration in a 79-year-old man who had previously been asymptomatic (Dumler, 1966).

On the basis of this experience, it is suggested that surgical transfer of the anomalous left pulmonary artery to relieve symptomatic airway obstruction should not be undertaken unless it is obvious that life is threatened. If tracheobronchial stenosis is present, operative division of the aberrant vessel is unlikely to produce a significant improvement in symptoms. There have been considerable advances in respiratory care for infants in recent years, which should allow more severely distressed patients to survive without operative intervention. With growth, symptoms become less even though the anomalous pulmonary artery remains in its abnormal position.

\section{References}

Dumler, M. P. (1966). A rare cause of dysphagia. Journal of the American Medical Association, 197, 513-514.

Grover, F. L., Norton, J. B., Webb, G. E., and Tinkle, J. K. (1975). Pulmonary sling. Case report and collective review. Journal of Thoracic and Cardiovascular Surgery, 69, 295-300.

Hiller, H. G., and Maclean, A. D. (1957). Pulmonary artery ring. Acta Radiologica, 48, 434-438.

Kale, M. K., Rafferty, R. E., and Carton, R. W. N (1970). Aberrant left pulmonary artery presenting o as a mediastinal mass. Archives of Internal $N$ Medicine, 125, 121-125.

Koopot, R., Nikaidoh, H., and Idriss, F. S. (1975). Surgical management of anomalous left pulmonary artery causing tracheobronchial obstruction. Pul- $Q$ monary artery sling. Journal of Thoracic and $\mathbb{\varnothing}$ Cardiovascular Surgery, 69, 239-246.

Lochard, J., Vert, P., and Chalnot, P. (1963). Trajet 0 aberrant de l'artère pulmonaire gauche comprimant $\bar{O}$ l'origine de la bronche souche droite. Annales de $\mathbb{\mathbb { D }}$ Chirurgie Thoracique et Cardio-Vasculaire, 2, 458461.

Miller, R. D., and Hyatt, R. E. (1973). Evaluation of obstructing lesions of the trachea and larynx by 
flow-volume loops. American Review of Respiratory Disease, 108, 475-481.

Philp, T., Sumerling, M. D., Fleming, J., and Grainger, R. G. (1972). Aberrant left pulmonary artery. Clinical Radiology, 23, 153-159.

Sade, R. M., Rosenthal, A., Fellows, K., and Castaneda, A. R. (1975). Pulmonary artery sling. Journal of Thoracic and Cardiovascular Surgery, 69, 333-346.
Tesler, U. F., Balsara, R. H., and Niguidula, F. N. (1974). Aberrant left pulmonary artery (vascular sling): report of 5 cases. Chest, 66, 402-407.

Requests for reprints to: Dr P. D. Phelan, Director, Department of Thoracic Medicine, Royal Children's Hospital, Flemington Road, Parkville, Melbourne, Victoria 3052, Australia. 